\title{
Korea-Australia Rheology Journal
}

\author{
Co- Editors in Chief \\ Hyun Wook Jung \\ Department of Chemical and Biological Engineering, Korea University, Seoul, Republic of Korea \\ hwjung@grtrkr.korea.ac.kr \\ Ravi P. Jagadeeshan \\ Dept. of Chemical Engineering, Monash University, Australia \\ Ravi.Jagadeeshan@monash.edu
}

\section{Accepted February $19^{\text {th }} 2018$}

In press

ISSN: 1226-119X (print version)

ISSN: 2093-7660 (electronic version)

Impact Factor: 1.000

\section{ELECTROOSMOTIC FLOW OF BIORHEOLOGICAL MICROPOLAR FLUIDS THROUGH MICROFLUIDIC CHANNELS}

\author{
M.K. Chaube', A. Yadav², D. Tripathi ${ }^{2 *}$ and O. Anwar Bég \\ ${ }^{1}$ DSPM IIIT Naya Raipur,Chhattisgarh-493661, India. \\ ${ }^{2}$ Department of Mechanical Engineering, Manipal University Jaipur, Rajasthan--303007, India. \\ ${ }^{3}$ Fluid Mechanics and Propulsion, Department of Mechanical and Aeronautical Engineering, \\ Salford University, Newton Building, The Crescent, Salford, M54WT, England, UK.
}

\begin{abstract}
An analytical analysis is presented in this work to assess the influence of micropolar nature of fluids in fully developed flow induced by electrokinetically driven peristaltic pumping through a parallel plate microchannel. The walls of the channel are assumed as sinusoidal wavy to analyze the peristaltic flow nature. We consider that the wavelength of the wall motion is much larger as compared to the channel width to validate the lubrication theory. To simplify the Poisson Boltzmann equation, we also use the Debye-Hückel linearization (i.e. wall zeta potential $\leq 25 \mathrm{mV}$ ). We consider governing equation for micropolar fluid in absence of body force and couple effects however external electric field is employed. The solutions for axial velocity, spin velocity, flow rate, pressure rise and stream functions subjected to given physical boundary conditions are computed. The effects of pertinent parameters like Debye length and HelmholtzSmoluchowski velocity which characterize the EDL phenomenon and external electric field, coupling number and micropolar parameter which characterize the micropolar fluid behavior, on peristaltic pumping are discussed through the illustrations. The results show that peristaltic pumping may alter by applying external electric fields. This model can be used to design and engineer the peristalsis-lab-on-chip and micro peristaltic syringe pumps for biomedical applications.
\end{abstract}

Keywords: Electroosmosis; Electric double layer; Peristalsis; Microrotation; Mechanical efficiency; Trapping.

“*Corresponding Author, E-mail: dharmendra.tripathi@jaipur.manipal.edu” 


\section{INTRODUCTION}

Electrokinetics or Electro-fluid-dynamics (EFD) is the study of the dynamics of electrically charged fluids which is also defined as a bulk movement of the liquid relative to a stationary surface subjected to an applied external electric field. It is applicable in a wide variety of developing technologies, extending from the cooling of electronic devices to biomedical diagnostics. It has gradually emerged as a more preferred alternative flow mechanism for transporting the fluids through microfluidic channels/tubes since there is an absence of accurate control mechanisms currently available for typical pressure-driven microflows. It can be used to fulfill the pumping power requirements and also integrated with the peristalsis-lab-on-chip. In this direction, an analytical approach for electrokinetic flow through capillary slits was presented subjected to Debye-Hückel linearization by Burgreen and Nakache [1]. Later this study was discussed numerically for a narrow cylindrical capillary by Rice and Whitehead [2] and numerical results were also validated with approximate solutions. This study was further modified for high zeta potential by Levine et al. [3]. In some other studies [4-6], applications of electrokinetic effect were discussed. Recently, Obliger et al. [7] presented a pore network model of electrokinetic transport through charged porous media. Chakraborty [8] discussed the applications of electrokinetic transport in Biochemical Reactions. Most recently, some investigations [9-14] on electrokinetic transport and its applications are reported in literature in which electrokinetic transport with non-Newtonian fluids, nano channels, thermally developing MHD flow, thermal transport and monovalent and divalent cations in silica are studied.

In addition of electrokinetic, peristalsis is also taken into consideration in this study to focus the applications in physiological transports. Peristalsis is a natural phenomenon specially found in digestive system to propel the food from mouth to stomach and chyme from stomach to large intestine via small intestine. It is also similar to heart pump by which blood is taken to every part of body. It is also observed in urine flow from the kidney to the bladder, flow of spermatozoa in the ductus efferentes of the male reproductive tract, movement of ovum in the female fallopian tube, and transport of lymph in the lymphatic vessels. Considering the biomedical importance of peristaltic pumping of non-Newtonian fluids like Jeffrey fluid [15], sisko fluid [16], Jeffrey nanofluid [17] and Carreau fluid [18], 
some theoretical investigations have recently been presented. Using the combined effects of electroosmosis and peristaltic pumping, [19] have reported first model where he discussed the enhancement of peristaltic pumping by electroosmotic mechanism. He also considered the thin electric double layer (EDL) where effects of charged surface i.e. EDL phenomenon is assumed negligible. Sequentially improvements in this study are reported recently in few investigations [20-23] where EDL effects, MHD effects, power law fluids and couple stress fluids are taken into consideration.

These studies also concluded that peristaltic pumping can be enhanced with applied external electric field. However there is no study on micropolar fluids flow driven by electro-osmotic modulated peristaltic pumping. Micropolar fluid represents the fluid which consist the randomly oriented particles suspended in a viscous medium. It can support couple stresses, body couples and exhibits micro-rotational and micro-inertial effects. It can be applicable in physiological fluids transport where suspension of particles play import role like in blood (suspension of $\mathrm{RBC}, \mathrm{WBC}$ and platelets). The theory of micropolar fluid was first initiated by Eringen [24]. And in this direction, some interesting studies [25-29] on micropolar fluids with peristaltic pumping are documented. The effects of coupling parameter and micropolar parameter on peristaltic flow characteristics are discussed. Recently some few investigations [30-35] on micropolar fluids flow induced by electrokinetic transport are presented where the effect of coupling parameter and micropolar parameter on electro-osmosis are examined. With regard to the rheological focus of the current work, it is pertinent to mention that many biochemical analytical systems in reality are frequently used to process biofluids, such as saliva solutions, DNA solutions, and blood samples, and these cannot be simulated as Newtonian fluids. These systems exhibit strong non-Newtonian behaviour which has been confirmed experimentally by for example Olivares et al. [36] who showed that electrokinetic phenomena involving non-Newtonian fluids strongly deviate from their Newtonian counterparts. They reported extensive experimental investigations for the electro-osmotic flow of a typical polymer solution in microchannels showing that the fluids involved follow closely viscoelastic and microstructural models of rheology owing to their very complex mciro-rheology. References [37] and [38] reported rheological electrokinetic phenomena due to the viscoelectric effect and utilized rheological models in which viscosity of the 
fluids is a quadratic function of the local electric field strength. These studies also demonstrated that the electrokinetic mobility in shear thinning fluids in laboratory experiments is significantly greater than that observed in Newtonian fluids. The electroosmotic flow is progressively suppressed with subsequent electrophoretic runs, suggesting a "dynamic coating" of the polymers onto the capillary wall. Owing to the very high shear rate in the electric double layer, the polymer molecules change their orientation and/or conformation, which reduces fluid viscosity in this region. These investigations [3638] have also emphasized that viscosity of polyelectrolyte solutions is very intimately associated with micro-particles and suspensions which in turn are dependent on the solvent quality and the polymer concentration regime. In this regard these studies imply that microstructure may exert a critical role in rheological characteristics of electro-osmotic flows in micro-channel designs. Further studies confirming the importannce of biorheological characterization of electro-osmotic micro-channel flows include [39, 40].

The fundamental aim of the present work is to theoretically analyze the influence of micropolar parameter and coupling parameter on peristaltically developing electroosmotic transport in parallel plate micro-channels. The flow is taken to be actuated by an axial electric field and peristaltic pumping. The zeta potential is considered less than $25 \mathrm{mV}$ so that Debye-Hückel approximation can be used. The wavelength of peristaltic wave is assumed to be greater than channel width. Closed form expressions are obtained subjected to physical constraints. The effect of relevant parameters on flow characteristics are numerically discussed with the help of illustrations.

\section{MATHEMATICAL FORMULATION OF PROBLEM}

We consider the electroosmotic-augmented peristaltic transport of an incompressible micropolar fluid through a microfluidic channel of width $2 a$. Let $Y= \pm H$ be the upper and lower boundaries of the channel. The motion is considered to be induced by sinusoidal wave trains propagating along the channel walls with a constant speed $c$. The schematic diagram of the problem under consideration is depicted in Figure 1 and mathematically considered as: 


$$
H=a+b \sin \frac{2 \pi}{\lambda}\left(X^{\prime}-c t^{\prime}\right)
$$

where $b$ is the wave amplitude, $\lambda$ is the wave length and $t^{\prime}$ is the time. The flow is unsteady in the laboratory frame $\left(X^{\prime}, Y^{\prime}\right)$, whereas it is steady if observed in the coordinate system $\left(x^{\prime}, y^{\prime}\right)$, termed as wave frame, moving with the wave speed $c$. The transformations between these two coordinate systems are as follows:

$$
x^{\prime}=X^{\prime}-c t^{\prime}, \quad y^{\prime}=Y^{\prime}, u^{\prime}=U^{\prime}-c, v^{\prime}=V^{\prime},
$$

which $\left(u^{\prime}, v^{\prime}\right)$ and $\left(U^{\prime}, V^{\prime}\right)$ are the velocity components in the wave and laboratory frames of reference respectively.

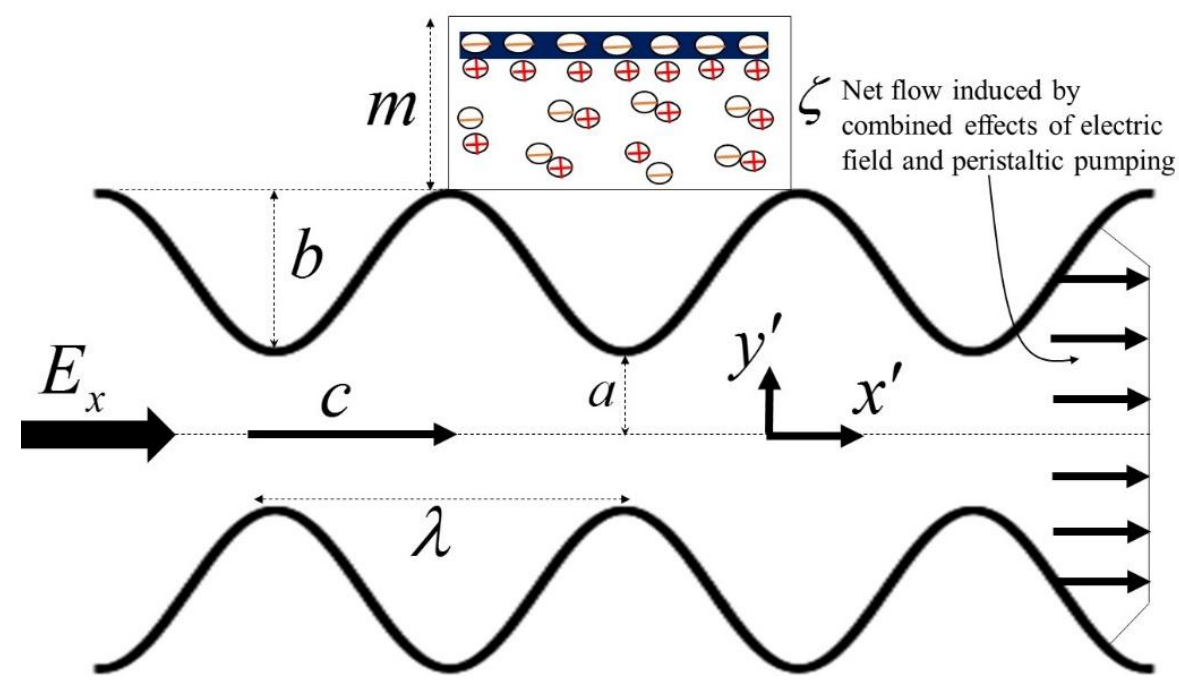

Figure 1. Schematic diagram of electroosmotic flow in presence of peristaltic wave propulsion with wave velocity $c$ and wavelength $\lambda$.

In the absence of body forces and the body couple, the governing equations for the steady flow of an incompressible micropolar fluid driven by combined effects of peristaltic pumping and electroosmosis, are given by (see Refs. [26-31])

$$
\begin{gathered}
\frac{\partial u^{\prime}}{\partial x^{\prime}}+\frac{\partial v^{\prime}}{\partial y^{\prime}}=0 \\
\rho\left(u^{\prime} \frac{\partial u^{\prime}}{\partial x^{\prime}}+v^{\prime} \frac{\partial u^{\prime}}{\partial y^{\prime}}\right)=-\frac{\partial p^{\prime}}{\partial x^{\prime}}+(\mu+\kappa)\left(\frac{\partial^{2} u^{\prime}}{\partial x^{\prime 2}}+\frac{\partial^{2} u^{\prime}}{\partial y^{\prime 2}}\right)+\kappa \frac{\partial w^{\prime}}{\partial y^{\prime}}+\rho_{e} E_{x},
\end{gathered}
$$




$$
\begin{gathered}
\rho\left(u^{\prime} \frac{\partial v^{\prime}}{\partial x^{\prime}}+v^{\prime} \frac{\partial v^{\prime}}{\partial y^{\prime}}\right)=-\frac{\partial p^{\prime}}{\partial y^{\prime}}+(\mu+\kappa)\left(\frac{\partial^{2} v^{\prime}}{\partial x^{\prime 2}}+\frac{\partial^{2} v^{\prime}}{\partial y^{\prime 2}}\right)-\kappa \frac{\partial w^{\prime}}{\partial x^{\prime}}, \\
\rho J^{\prime}\left(u^{\prime} \frac{\partial w^{\prime}}{\partial x^{\prime}}+v^{\prime} \frac{\partial w^{\prime}}{\partial y^{\prime}}\right)=-2 \kappa w^{\prime}+\gamma\left(\frac{\partial^{2} w^{\prime}}{\partial x^{\prime 2}}+\frac{\partial^{2} w^{\prime}}{\partial y^{\prime 2}}\right)+\kappa\left(\frac{\partial v^{\prime}}{\partial x^{\prime}}-\frac{\partial u^{\prime}}{\partial y^{\prime}}\right),
\end{gathered}
$$

where $u^{\prime}$ and $v^{\prime}$ are the velocity components in the $x^{\prime}$ and $y^{\prime}$ directions respectively, $\rho$ is the density of the fluid, $p^{\prime}$ is the pressure, $w^{\prime}$ is the microrotation velocity components in the direction normal to both the $x^{\prime}$ and $y^{\prime}$ axes, $J^{\prime}$ is the micro-inertia constant, $\mu$ is the viscosity constant of the classical fluid dynamics, $\kappa, \gamma$ are the viscosity constants for micropolar fluid, $E_{x}$ is the external electric field.

Poisson's equation to describe the electric potential distribution for a symmetric $(z: z)$ binary electrolyte solution $\left(\mathrm{Na}^{+} \mathrm{Cl}^{-}\right)$, is expressed as:

$$
\nabla^{2} \Phi^{\prime}=-\frac{\rho_{e}}{\varepsilon}
$$

in which $\varepsilon$ is the permittivity and $\rho_{e}$ is the density of the total ionic charges given by, $\rho_{e}=e z\left(n^{+}-n^{-}\right)$, in which $n^{+}$and $n^{-}$are the number of densities of cations and anions respectively. For further analysis, we use the following non-dimensional variables and parameters:

$$
\begin{gathered}
x=\frac{x^{\prime}}{\lambda}, y=\frac{y^{\prime}}{a}, u=\frac{u^{\prime}}{c}, v=\frac{v^{\prime}}{\delta c}, \delta=\frac{a}{\lambda}, w=\frac{a w^{\prime}}{c}, J=\frac{J^{\prime}}{a^{2}}, \\
h=\frac{H}{a} \Phi=\frac{\Phi^{\prime}}{\zeta}, p=\frac{a^{2} p^{\prime}}{c \lambda \mu}, \phi=\frac{b}{a}, \operatorname{Re}=\frac{\rho c a}{\mu}
\end{gathered}
$$

in which $\operatorname{Re}, \delta$ and $\zeta$ represent the Reynolds number, wave number and zeta potential respectively. Employing the non-dimensional variables in Eqs.(3-6), we get:

$$
\begin{gathered}
\frac{\partial u}{\partial x}+\frac{\partial v}{\partial y}=0 \\
\operatorname{Re} \delta\left(u \frac{\partial u}{\partial x}+v \frac{\partial u}{\partial y}\right)=-\frac{\partial p}{\partial x}+\left(\frac{\mu+\kappa}{\mu}\right)\left(\delta^{2} \frac{\partial^{2} u}{\partial x^{2}}+\frac{\partial^{2} u}{\partial y^{2}}\right)+\frac{\kappa}{\mu} \frac{\partial w}{\partial y}+m^{2} \Phi U_{H S} \\
\operatorname{Re} \delta^{3}\left(u \frac{\partial v}{\partial x}+v \frac{\partial v}{\partial y}\right)=-\frac{\partial p}{\partial y}+\delta^{2}\left(\frac{\mu+\kappa}{\mu}\right)\left(\delta^{2} \frac{\partial^{2} v}{\partial x^{2}}+\frac{\partial^{2} v}{\partial y^{2}}\right)-\delta^{2} \frac{\kappa}{\mu} \frac{\partial w}{\partial x}
\end{gathered}
$$




$$
\rho a c J \delta\left(u \frac{\partial w}{\partial x}+v \frac{\partial w}{\partial y}\right)=-2 \kappa w+\frac{\gamma}{a^{2}}\left(\delta^{2} \frac{\partial^{2} w}{\partial x^{2}}+\frac{\partial^{2} w}{\partial y^{2}}\right)+\kappa\left(\delta^{2} \frac{\partial v}{\partial x}-\frac{\partial u}{\partial y}\right) .
$$

where $N=\kappa /(\mu+\kappa)$ is the coupling number $(0 \leq N \leq 1), M^{2}=a^{2} \kappa(2 \mu+\kappa) /(\gamma(\mu+\kappa))$ is the micropolar parameter, $m=a e z \sqrt{\frac{2 n_{0}}{\varepsilon K_{B} T}}$, is known as the electro-osmotic parameter (inverse of Debye length) in which $K_{B}$ is the Boltzmann constant, $T$ is the average temperature of the electrolytic solution and $U_{H S}=-\frac{E_{x} \varepsilon \zeta}{\mu c}$ is the dimensionless HelmholtzSmoluchowski velocity. Applying lubrication approximation $(\delta=a / \lambda \square 1)$, the governing equations take the following form:

$$
\begin{gathered}
\frac{\partial^{2} u}{\partial y^{2}}+N \frac{\partial w}{\partial y}+(1-N) m^{2} \Phi U_{H S}=(1-N) \frac{\partial p}{\partial x} \\
\frac{\partial p}{\partial y}=0 \\
-2 w-\frac{\partial u}{\partial y}+\left(\frac{2-N}{M^{2}}\right) \frac{\partial^{2} w}{\partial y^{2}}=0
\end{gathered}
$$

Applying the Debye-Hückel linearization $(\zeta \leq 25 \mathrm{mV})$, the Poisson-Boltzmann equation reduces to:

$$
\frac{\partial^{2} \Phi}{\partial y^{2}}=m^{2} \Phi
$$

In the laboratory frame, the dimensional volume flow rate is defined as:

$$
Q\left(X^{\prime}, t^{\prime}\right)=\int_{0}^{H} U^{\prime}\left(X^{\prime}, Y^{\prime}, t^{\prime}\right) d Y^{\prime}
$$

where $H=H\left(X^{\prime}, t^{\prime}\right)$, which, in the wave frame, may be expressed as:

$$
q\left(x^{\prime}\right)=\int_{0}^{H} u^{\prime}\left(x^{\prime}, y^{\prime}\right) d y^{\prime},
$$

where $H=H\left(x^{\prime}\right)$. Eqs. (2), (17) and (18) yield:

$$
Q\left(X^{\prime}, t^{\prime}\right)=q\left(x^{\prime}\right)+c H\left(X^{\prime}, t^{\prime}\right) \text {. }
$$

The time-averaged flow rate over a time period $T$ at a fixed position $X^{\prime}$ is given by 


$$
\bar{Q}\left(X^{\prime}\right)=\frac{1}{T} \int_{0}^{T} Q\left(X^{\prime}, t^{\prime}\right) d t^{\prime}
$$

Using Eq. (19) into Eq. (20) we get

$$
\bar{Q}\left(X^{\prime}\right)=q\left(x^{\prime}\right)+a c,
$$

which is in nondimensional form expressed as:

$$
Q(X)=q(x)+1 .
$$

The boundary conditions are imposed as:

$$
\begin{gathered}
u(x, y)=-1 \text { at } y= \pm h(x), \\
w(x, y)=0 \quad \text { at } y= \pm h(x), \\
\frac{\partial \Phi}{\partial y}=0 \text { at } y=0, \Phi=1 \text { at } y=h(x) .
\end{gathered}
$$

\section{ANALYTICAL SOLUTIONS}

Solving Eq.(16) and using boundary conditions (25), the potential function is obtained as:

$$
\Phi=\frac{\cosh (m y)}{\cosh (m h)} .
$$

Solving simultaneous partial differential Eqs.(13) and (15), with boundary conditions (23) and (24), the axial and spin velocities are obtained respectively as:

$$
\begin{gathered}
u=\frac{N(1-N)(\cosh (M h)-\cosh (M y))}{M(2-N) \sinh (M h)}\left(h \frac{d p}{d x}+\frac{M^{2} m}{m^{2}-M^{2}} \tanh (m h) U_{H S}\right)+ \\
\left(\frac{1-N}{2-N}\right)\left(y^{2}-h^{2}\right) \frac{d p}{d x}+\left(\frac{1-N}{m^{2}-M^{2}}\right)\left(\frac{2 M^{2}}{2-N}-m^{2}\right)\left(\frac{\cosh (m y)}{\cosh (m h)}-1\right) U_{H S}-1, \\
w=\left(\frac{1-N}{2-N}\right)\left(\frac{h \sinh (M y)}{\sinh (M h)}-y\right) \frac{d p}{d x}+\left(\frac{1-N}{2-N}\right)\left(\frac{M^{2} m}{m^{2}-M^{2}}\right) \\
\left(\frac{\tanh (m h) \sinh (M y)}{\sinh (M h)}-\frac{\sinh (m y)}{\cosh (m h)}\right) U_{H S} .
\end{gathered}
$$

The stream function $(\psi)$ is defined as $u=\frac{\partial \psi}{\partial y}, v=-\frac{\partial \psi}{\partial x}$ and substituting $u=\frac{\partial \psi}{\partial y}$ in Eq.(27), we get 


$$
\begin{gathered}
\psi=\frac{N(1-N)(y \cosh (M h)-\sinh (M y) / M)}{M(2-N) \sinh (M h)}\left(h \frac{d p}{d x}+\frac{M^{2} m}{m^{2}-M^{2}} \tanh (m h) U_{H S}\right)+ \\
\left(\frac{1-N}{2-N}\right)\left(\frac{y^{3}}{3}-h^{2} y\right) \frac{d p}{d x}+\left(\frac{1-N}{m^{2}-M^{2}}\right)\left(\frac{2 M^{2}}{2-N}-m^{2}\right)\left(\frac{\sinh (m y)}{m \cosh (m h)}-y\right) U_{H S}-y .
\end{gathered}
$$

The dimensionless flow rate, in the wave frame of reference, is obtained a

$$
\begin{aligned}
q= & \frac{N(1-N)(h \cosh (M h)-\sinh (M h) / M)}{M(2-N) \sinh (M h)}\left(h \frac{d p}{d x}+\frac{M^{2} m}{m^{2}-M^{2}} \tanh (m h) U_{H S}\right) \\
& -\frac{2}{3}\left(\frac{1-N}{2-N}\right) h^{3} \frac{d p}{d x}+\left(\frac{1-N}{m^{2}-M^{2}}\right)\left(\frac{2 M^{2}}{2-N}-m^{2}\right)\left(\frac{\tanh (m h)}{m}-h\right) U_{H S}-h .
\end{aligned}
$$

Rearranging the terms in Eq.(30), the pressure gradient is derived as:

$$
\frac{d p}{d x}=\frac{(2-N)\left(q+h-f_{2}(h)-f_{3}(h)\right)}{(1-N) f_{1}(h)},
$$

where,

$$
\begin{gathered}
f_{1}(h)=-\frac{2}{3} h^{3}+\frac{N\left(h^{2} \cosh (M h)-h \sinh (M h) / M\right)}{M \sinh (M h)}, \\
f_{2}(h)=\frac{N(1-N) M^{2} m(h \cosh (M h)-\sinh (m h) / M) \tanh (m h) U_{H S}}{M(2-N)\left(m^{2}-M^{2}\right) \sinh (M h)}, \\
f_{3}(h)=\left(\frac{1-N}{m^{2}-M^{2}}\right)\left(\frac{2 M^{2}}{2-N}-m^{2}\right)\left(\frac{\tanh (m h)}{m}-h\right) U_{H S} .
\end{gathered}
$$

The pressure rise per wavelength is defined as (Shapiro et al.[41]):

$$
\Delta p=\int_{0}^{1} \frac{d p}{d x} d x .
$$

All above analytical solutions can be reduced to the analytical solution presented in the Hayat et al. [42] for $U_{H S}=0$. That means that Hayat's model [42] is a particular case of the present model.

\section{MECHANICAL EFFICIENCY}

Mechanical efficiency is defined as the ratio between the average rate per wavelength at which work is done by the moving fluid against a pressure head and the average rate at which the wall do work on the fluid (Shapiro et al. [41]). The mechanical efficiency for electroosmotic induced peristaltic transport of micropolar fluid is obtained as: 


$$
E=-\frac{\bar{Q} \Delta p}{\phi \int_{0}^{1} \frac{d p}{d x} \sin (2 \pi x) d x}
$$

\section{RESULTS AND DISCUSSION}

In order to study that how the various physical parameters affect the electroosmotic modulated peristaltic pumping characteristic like axial velocity, pressure rise, mechanical efficiency and trapping, the analytical solutions are numerically computed and illustrated through the Figs. (2-6).

Figs.2(a-d) illustrates the effects of coupling number $(N=\kappa /(\mu+\kappa))$ which is valid for the range $0 \leq N \leq 1$, micropolar parameter $\left(M^{2}=a^{2} \kappa(2 \mu+\kappa) / \gamma(\mu+\kappa)\right)$, electro-osmotic parameter $m=a e z \sqrt{\frac{2 n_{0}}{\varepsilon K_{B} T}}$, which characterizes the electric double layer (EDL) thickness (i.e. for $m \rightarrow \infty$, the EDL is very thin which physically interpret that there is no effect of charged distribution of wall surface on fluid flow) and $U_{H S}=-\frac{E_{x} \varepsilon \zeta}{\mu c}$ is the HelmholtzSmoluchowski velocity which characterizes the effect of applied external electric field (if there is no electric field $U_{H S} \rightarrow 0$ and physical problem will become peristaltic flow of micropolar fluid through microchannel), on velocity profile. Velocity profiles are plotted between axial velocity versus transverse displacement for negative pressure gradient ( $\frac{d p}{d x}<0$ i.e. $p_{x}=-5$ ) and other parameters are fixed $\phi=0.6, x=1$. It is observed that the velocity profile for negative pressure gradient is parabolic towards transverse direction which validate our present model because it is trivial that velocity profile for negative pressure gradient is always parabolic. Fig.2a depicts the effects of electro-osmotic parameter $(m=1,2,3)$ on velocity profile and it is noted that the region of velocity profile expands with reducing the thickness of electric double layer (i.e increasing $m$ ). Fig.2b shows the effect of Helmholtz-Smoluchowski velocity $\left(U_{H S}=0,1,2\right)$ on velocity profile where the curve for $U_{H S}=0$ shows velocity for pure peristaltically driven flow (See Hayat et al. [42]). It is also observed that the velocity is increasing with increasing the magnitude 
of electric field that physically interprets that peristaltic flow can be enhanced by electroosmosis. Fig.2c represents the effect of micropolar parameter $(M=1,10,100)$ on velocity profile and it is revealed that with huge change (1-100) in magnitude of micropolar parameter, there is a slight increment in velocity profile that means the micropolar parameter does not much affect the flow characteristics. Fig.2d depicts the effect of coupling parameter $(N=0,0.2,0.4)$ where the curve for $N=0$ represents the velocity profile for Newtonian fluid. It is further inferred that the velocity profile diminishes with increasing the magnitude of coupling parameter. It is physically interpreted that the micropolar nature of fluids opposes the fluid flow.

The influences of coupling number, micropolar parameter, electro-osmotic parameter, and the Helmholtz-Smoluchowski velocity on the variation of pressure difference $(\Delta p)$ with time averaged flow rate $(\bar{Q})$ are seen through the Figs.3(a-d). The graphs are plotted between pressure difference $(\Delta p)$ and time averaged flow rate $(\bar{Q})$ to study the pumping characteristics at $\phi=0.6$. The relation between pressure difference and time averaged flow rate is found to be linear which is similar to results of Shapiro et al. [41]. It is also pointed out that the maximum flow rate $\left(\bar{Q}_{0}\right)$ is achieved at zero pressure difference $(\Delta p=0)$ and vice versa. On the basis of pressure difference there are three regions classified as pumping region for $\Delta p>0$, augmented pumping region for $\Delta p<0$ and free pumping for $\Delta p=0$. Fig.3a shows the effect of electro-osmotic parameter $(m=1,2,3)$ on pressure difference and it is reported that the pressure difference elevates with reducing the thickness of electric double layer (i.e. increasing the value of $m$ ) in all pumping regions. Fig.3b presents the effect of Helmholtz-Smoluchowski velocity $\left(U_{H S}=0,1,2\right)$ on pressure difference and it is observed that pressure difference enhances with increasing the electric field. The curve at $U_{H S}=0$ shows the pressure rise generated by purely peristaltic pumping which is same as the pressure rise obtained by Hayat et al. [42] for sinusoidal flow regime. Fig.3c illustrates the changes in pressure difference with micropolar parameter $(M=1,10,100)$ and it is pointed out that there is minor changes with increasing the magnitude of $M$, increment in augmented pumping region, reduction in pumping region and no changes at free pumping 
zone. Fig.3d reveals the impact of coupling parameter $(N=0,0.2,0.4)$ on pressure difference and it is noted that the effect of $N$ on pressure difference is opposite to that of $M$.

The variation of mechanical efficiency against ratio of time averaged flow rate $(\bar{Q})$ and maximum averaged flow rate $\left(\bar{Q}_{0}\right)$ are shown through the Figs.4(a-d) subjected to effects of various pertinent physical parameters at $\phi=0.8$. The ranges of $\bar{Q} / \bar{Q}_{0}$ is considered from 0 to 1 . It is inferred that the value of mechanical efficiency starts with zero at $\bar{Q} / \bar{Q}_{0}=0$ (it means zero flow rate) and linearly increases and attain maximum value in left neighborhood of $\bar{Q} / \bar{Q}_{0}=1$ (it means flow rate is very close to maximum flow rate) thereafter it goes down to zero at $\bar{Q} / \bar{Q}_{0}=1$ (it means the flow rate becomes maximum) which is very similar to the results for mechanical efficiency reported by Shapiro et al. [41]. The effect of electro-osmotic parameter $(m=1,2,3)$ on mechanical efficiency is seen in Fig.4a and mechanical efficiency increases with decreasing the EDL thickness. The influence of Helmholtz-Smoluchowski velocity $\left(U_{H S}=0,1,2\right)$ on mechanical efficiency is presented in Fig. $4 \mathrm{~b}$ and mechanical efficiency goes up with increasing the electric filed. The alterations in mechanical efficiency with micropolar parameter $(M=1,10,100)$ is depicted in Fig.4c and mechanical efficiency changes slightly (increasing) with a large change (increasing) in micropolar parameter. The reduction in mechanical efficiency with elevating the magnitude of coupling parameter $(N=0,0.2,0.4)$ is reported in Fig.4d.

An interesting phenomenon of peristaltic pumping known as trapping is discussed in two cases through Figs. 5(a-d) and Figs. 6(a-d) subjected to influences of coupling number, micropolar parameter and electro-osmotic parameter. Trapping is a process of recirculation of center stream lines at good combination of the values of averaged flow rate $(\bar{Q})$ and amplitude of peristaltic wave $(\phi)$. In this study the values of averaged flow rate and amplitude of peristaltic wave are considered as $\bar{Q}=0.6, \phi=0.5$. The first case (Figs.5(a-d)) is considered as for opposing the electric field $\left(U_{H S}<0\right)$ that means the electric filed is applied opposite to peristaltic pumping and second case (Figs.6(a-d)) is taken for adding 
the electric field $\left(U_{H S}>0\right)$ that means the electric field is applied towards the peristaltic flow direction. If we compare the adding and opposing the electric field on trapping, it is clear that the center stream lines recirculate and form the bolus in first case however the center lines are parallel and contracted at center of channel and adjacent lines recirculate in upper and lower parts of channel. It is physically interpreted that when peristaltic pumping is dominating (first case) then center lines form bolus while with combined effects of electric field and peristaltic pumping (second case) the center stream lines become straight and parallel and the stream lines towards channel walls are trapped which is due to EDL effects. The effect of micropolar parameter $(M=1,4)$ on trapping for first case is shown in figs.5a\&b. It is observed that with the changes from 1 to 4 in micropolar parameter, there are very minor changes (negligible) in trapping. The variation of coupling parameter $(N=0.1,0.5)$ is depicted in Figs.5a\&5c and it is revealed that size of trapping bolus reduces with change in $N$ from 0.1 to 0.5 . The effect of the alteration of electroosmotic parameter $(m=1,10)$ on trapping is illustrated in Figs5a $\& 5 \mathrm{~d}$ and it is found that the number of trapping boluses increases with a change of $m$ from 1 to 10. For second case (Figs.6(a-d)), the effects of all three parameters are similar to first case but in Fig.6c, there is no trapping bolus when we increase the magnitude of coupling parameter $N$ from 0.1 to 0.5 .

(a)

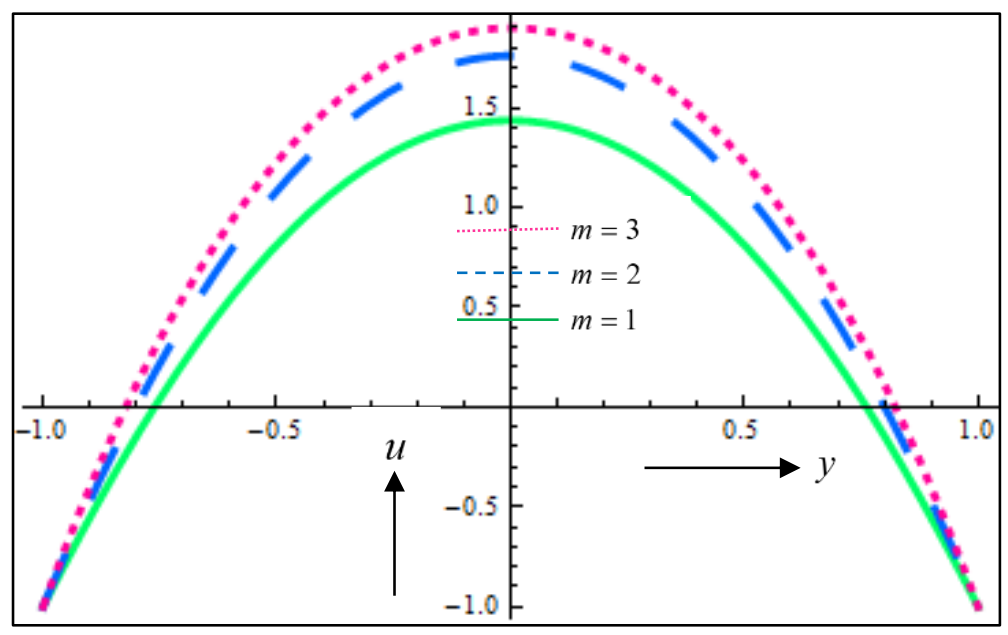


(b)

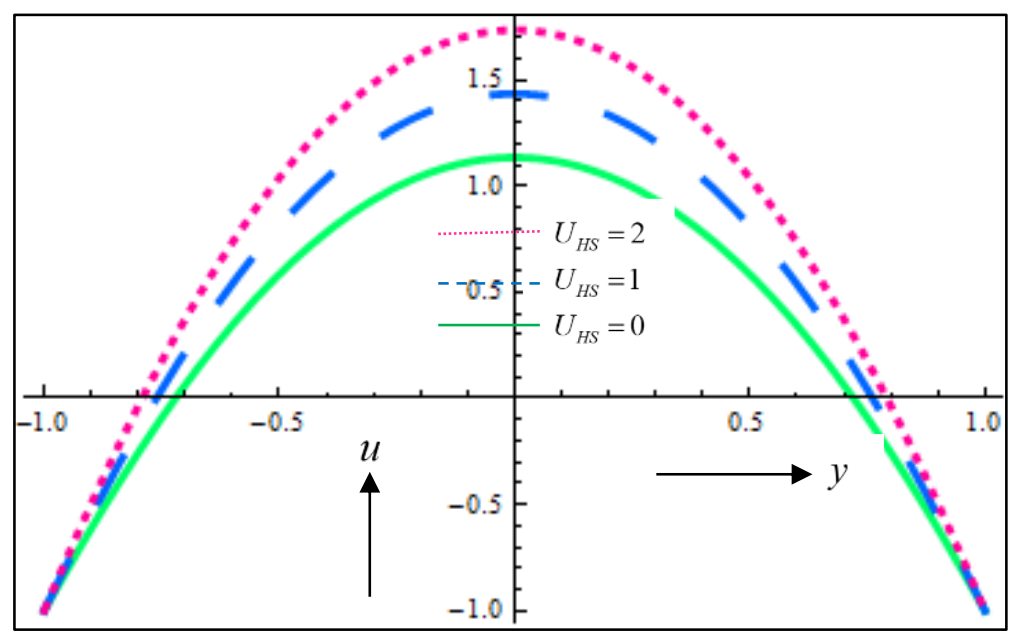

(c)

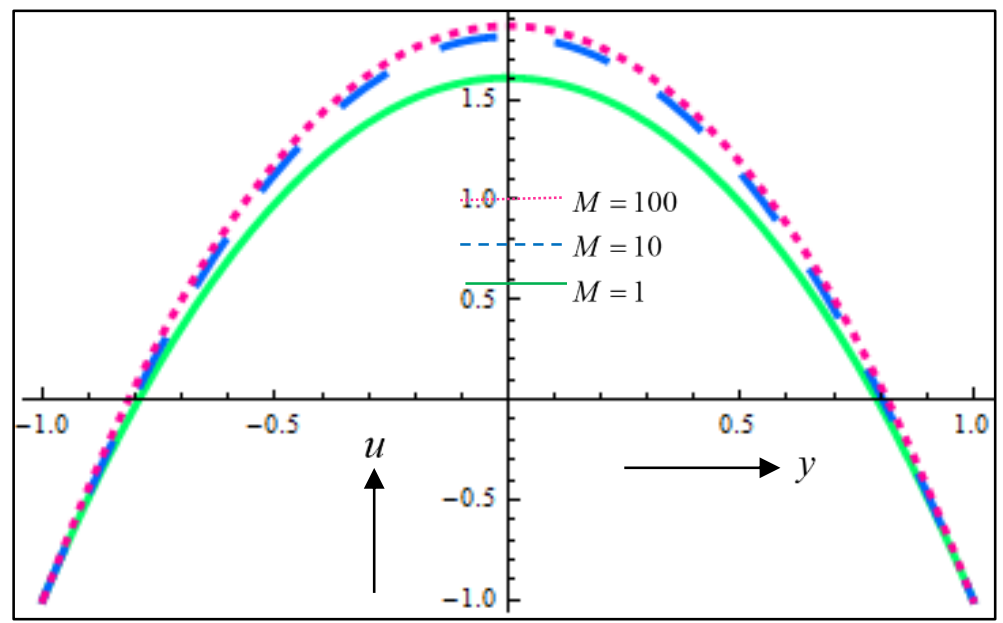

(d)

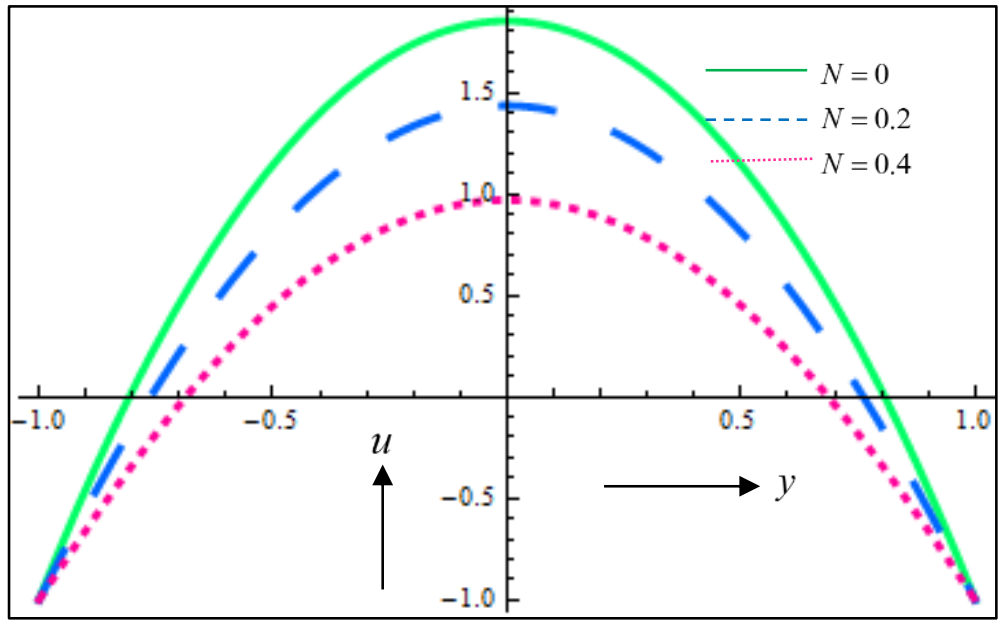

Fig.2. Velocity profile at $\phi=0.6, x=1, p_{x}=-5$ for different values (a) $m$ (b) $U_{H S}$ (c) $M(\mathrm{~d}) N$. 
(a)

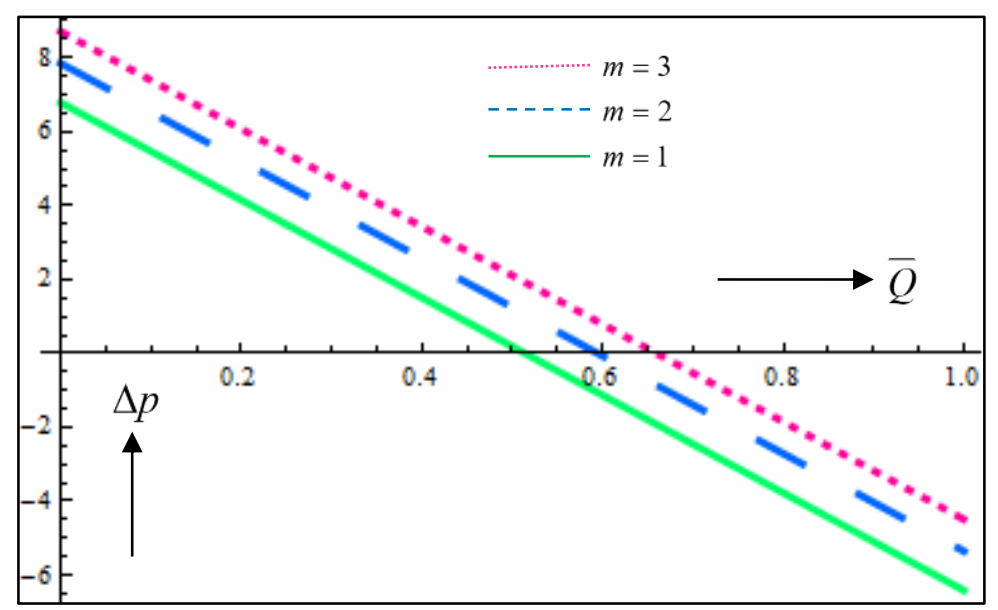

(b)

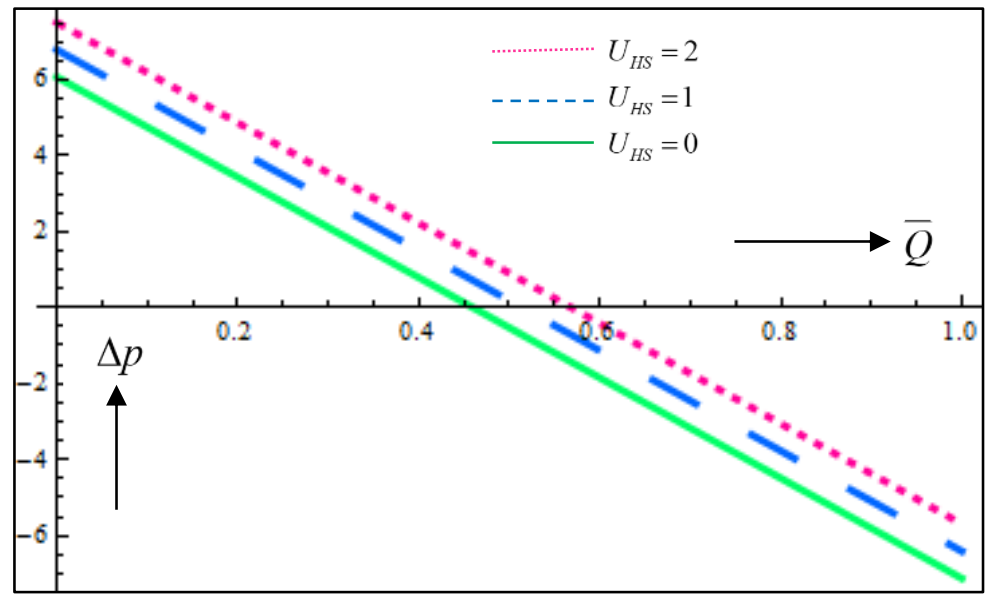

(c)

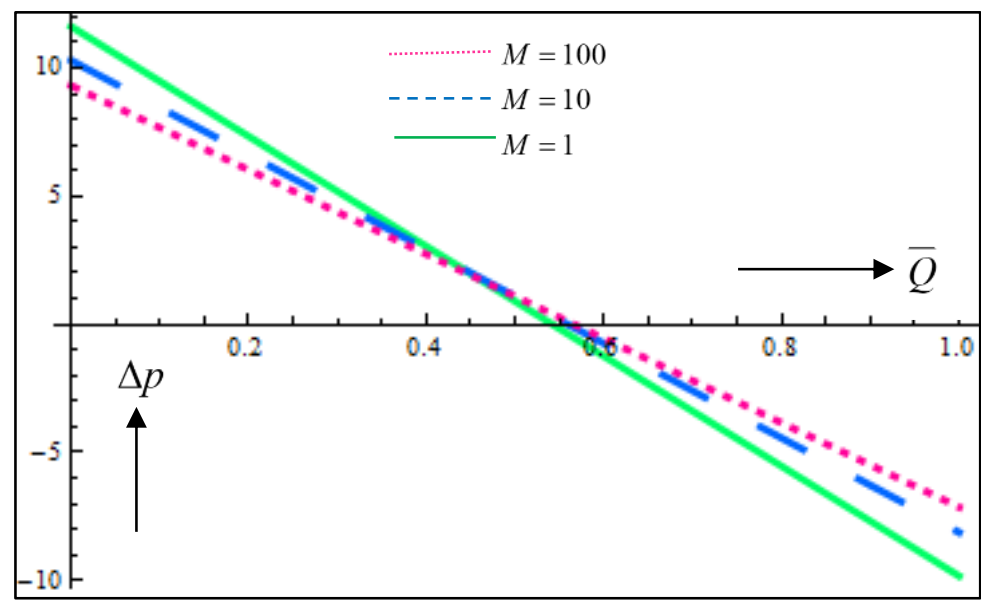


(d)

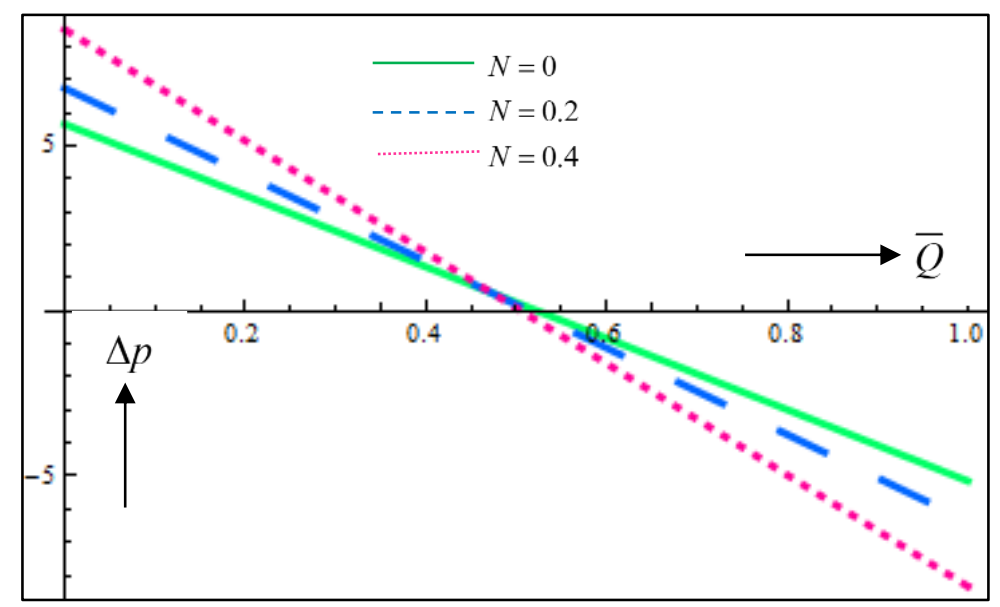

Fig.3. Plot between $\Delta p$ and $\bar{Q}$ at $\phi=0.6$ for different values (a) $m$ (b) $U_{H S}$ (c) $M$ (d) $N$.

(a)

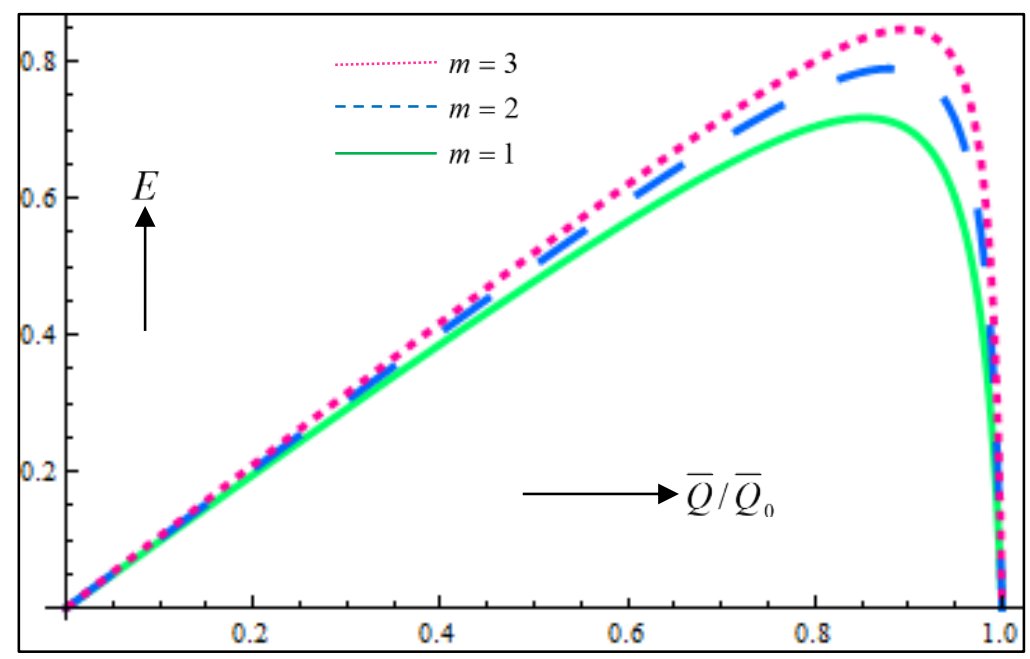

(b)

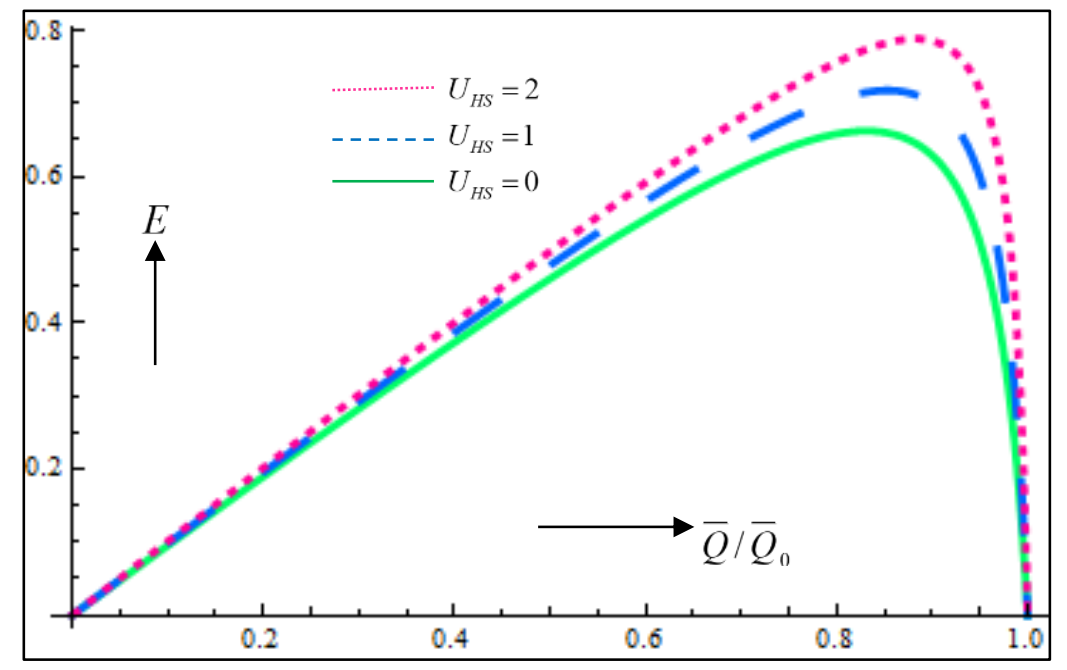


(c)

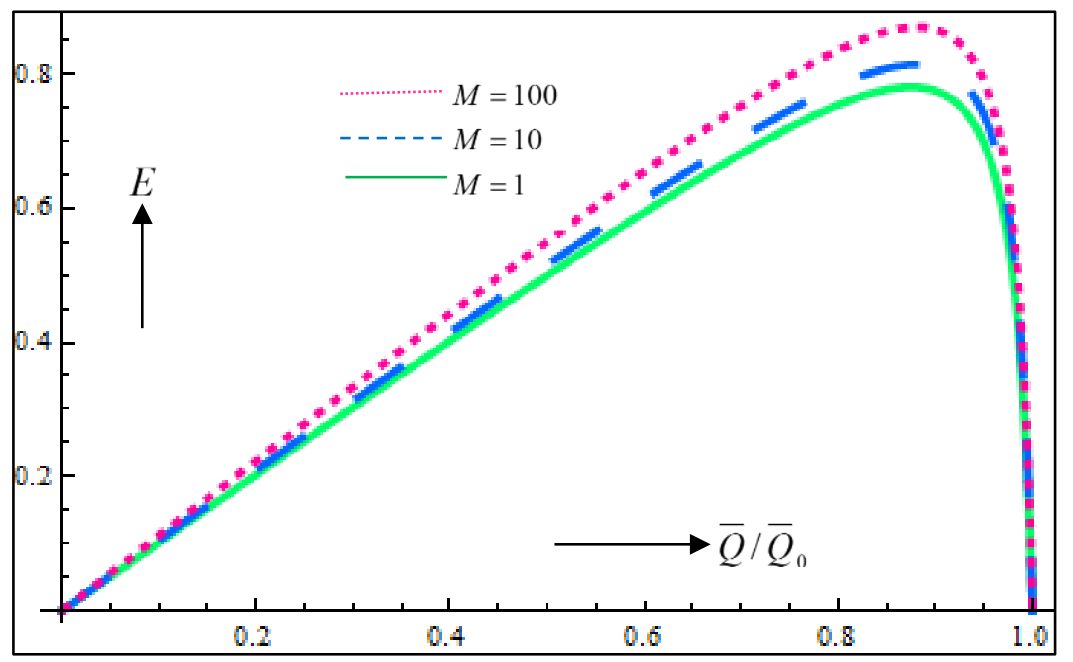

(d)

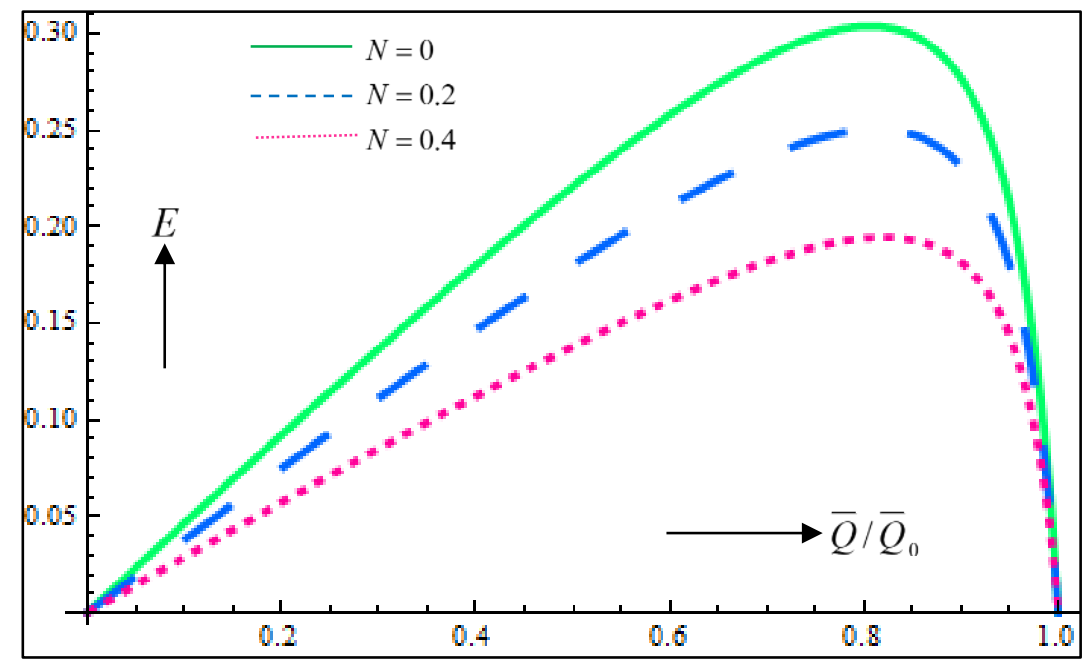

Fig.4. Mechanical efficiency vs. the ratio of averaged flow rate and maximum averaged flow rate at $\phi=0.8$ for different values of (a) $m$ (b) $U_{H S}$ (c) $M$ (d) $N$. 

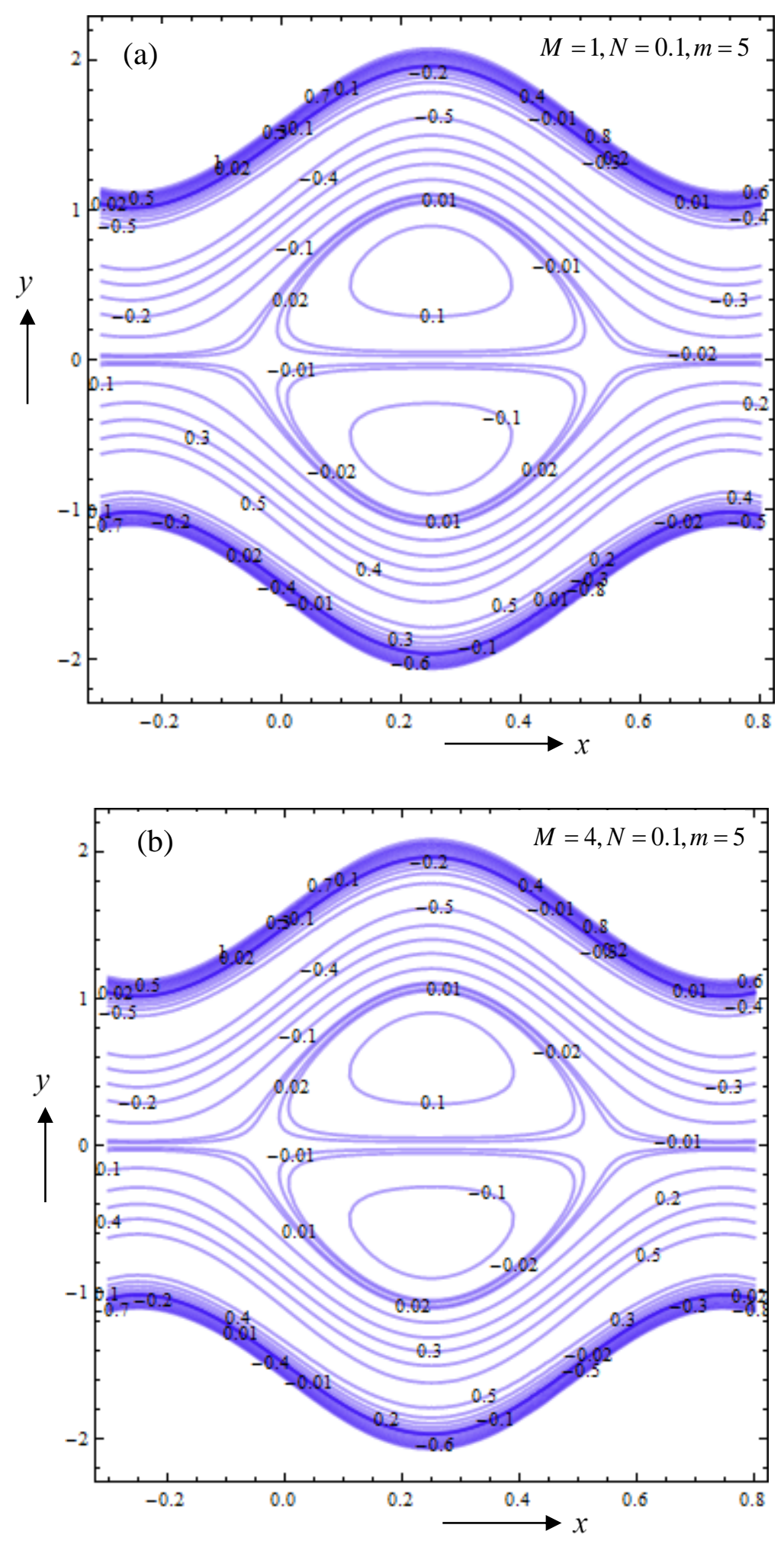

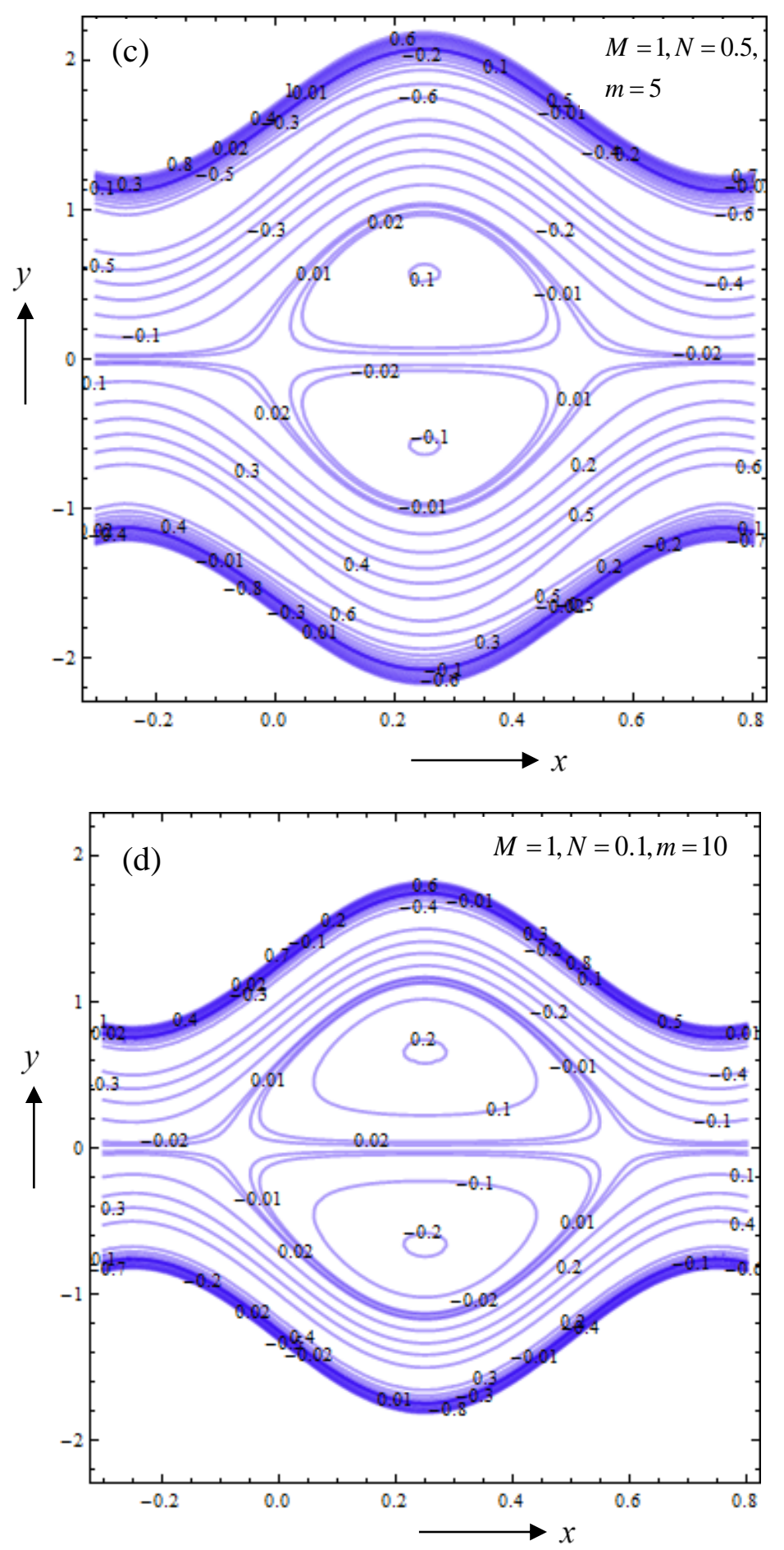

Fig.5. Stream lines at $\bar{Q}=0.6, \phi=0.5$ and $U_{H S}=-1$. 

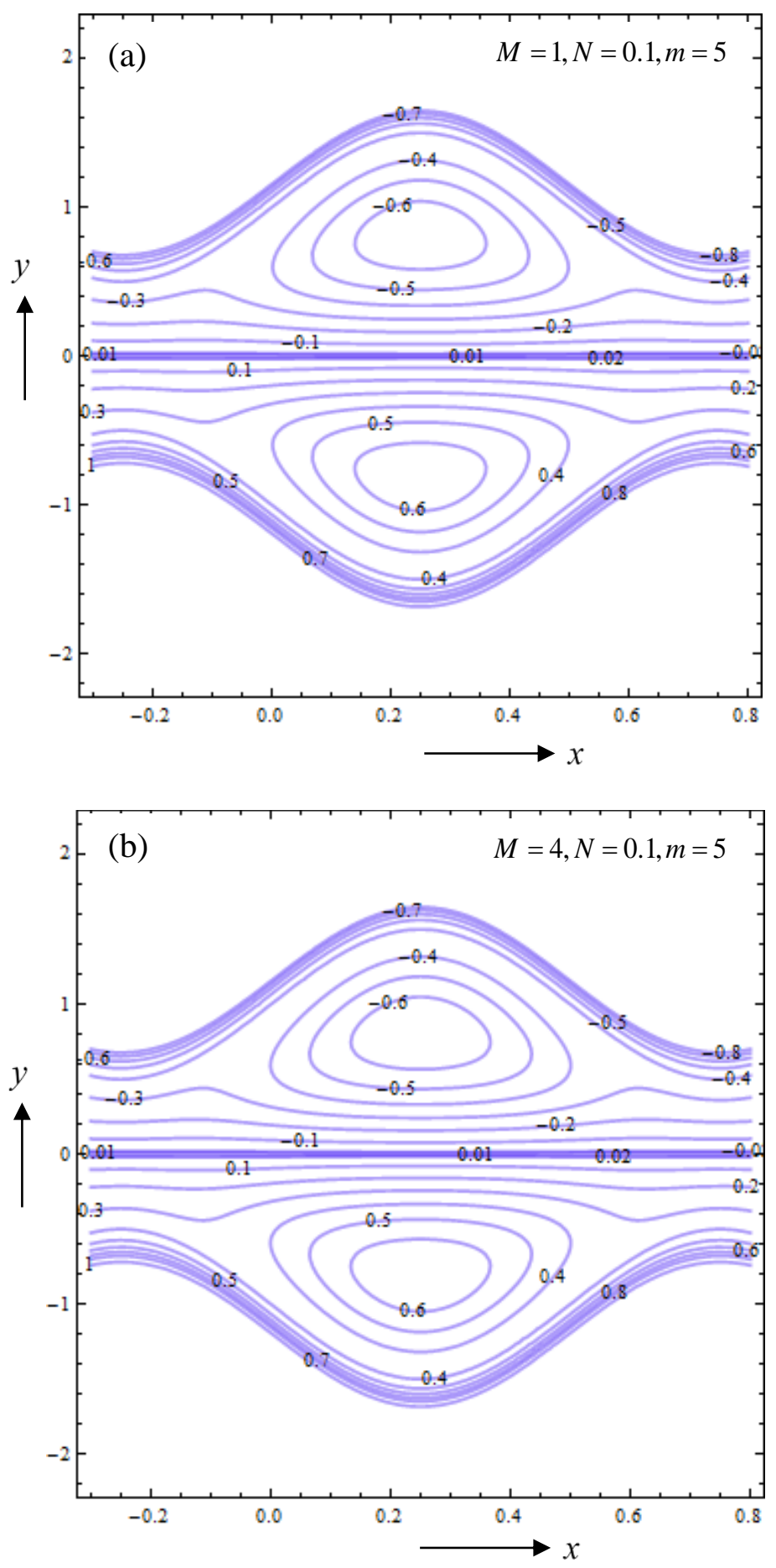

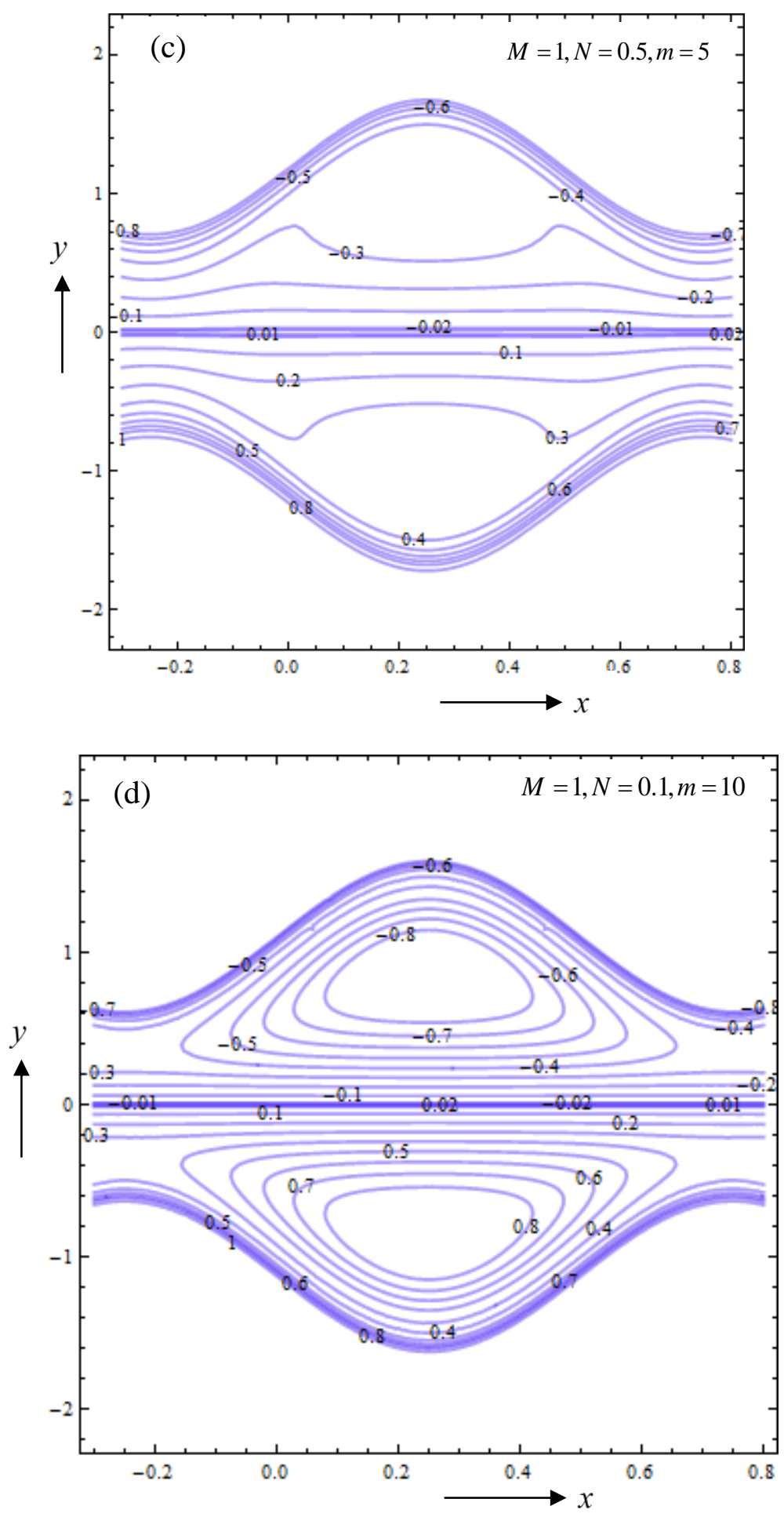

Fig.6. Stream lines at $\bar{Q}=0.6, \phi=0.5$ and $U_{H S}=5$. 


\section{CONCLUSIONS}

In this study, we analyze the electroosmosis modulated peristaltic transport of micropolar fluids through a microchannel. The effects of the coupling number, micropolar parameter, electro-osmotic parameter, and the Helmholtz-Smoluchowski velocity on velocity profile, pressure difference, mechanical efficiency and trapping phenomenon are discussed in the last section. The first observation of this study is that peristaltic transport of a micropolar fluid enhances with the applied external electric field and also alters with the electric double layer formation. Second observation of this study is that electroosmotic peristaltic flow varies with an increment in coupling parameter from 0 to 1 however there are minor changes (negligible) with large increment in magnitude of micropolar parameter from 1 to 100. The last and final observation of this study is that the center stream lines are trapped with the opposing electric field however the center stream lines are straight and parallel to channel length with the adding electric filed. This model is applicable to study the effects of microrotation of fluid particles (blood cells) during the fluids transport (blood flow). The findings of Hayat et al.[37] can be examined from present analysis with $U_{H S}=0$. The results may also be relevant to the physiological flows and industrial applications in which electroosmotic induced peristaltic pumping is used subjected to microrotation of fluid particles.

\section{References}

1. D Burgreen and Nakache F, The Journal of Physical Chemistry. 68, 1084 (1964)

2. C Rice and Whitehead R, The Journal of Physical Chemistry. 69, 4017 (1965)

3. S Levine, et al., Journal of Colloid and Interface Science. 52, 136 (1975)

4. E Bonaccurso, Kappl M, and Butt H-J, Physical Review Letters. 88, 076103 (2002)

5. M Z Bazant and Squires T M, Physical Review Letters. 92, 066101 (2004)

6. S J Kim, et al., Physical review letters. 99, 044501 (2007)

7. A Obliger, et al., Physical Review E. 89, 043013 (2014)

8. S Chakraborty, Encyclopedia of Microfluidics and Nanofluidics. 845 (2015)

9. A Bandopadhyay and Chakraborty S, Physical Chemistry Chemical Physics. 17, $7282(2015)$

10. S Prakash, et al., Microfluidics and Nanofluidics. 19, 1455 (2015) 
11. S Sarkar, Ganguly S, and Dutta P, International Journal of Heat and Mass Transfer. 100, 451 (2016)

12. M H Matin and Ohshima H, Journal of colloid and interface science. 476, 167 (2016)

13. S Prakash, et al., Microfluidics and Nanofluidics. 20, 8 (2016)

14. H Keramati, et al., International Journal of Heat and Mass Transfer. 92, 244 (2016)

15. Ellahi, R., M. Mubashir Bhatti, and Ioan Pop. International Journal of Numerical Methods for Heat \& Fluid Flow 26, no. 6 (2016): 1802-1820.

16. Bhatti, Muhammad Mubashir, A. Zeeshan, and R. Ellahi. Computers in Biology and Medicine 78 (2016): 29-41.

17. Bhatti, M. M., A. Zeeshan, and R. Ellahi. Microvascular Research 110 (2017): 3242.

18. Ellahi, R., M. M. Bhatti, and C. M. Khalique. Journal of Molecular Liquids 241 (2017): 1059-1068.

19. S Chakraborty, Journal of Physics D: Applied Physics. 39, 5356 (2006)

20. P Goswami, et al., Microvascular research. 103, 41 (2016)

21. D Tripathi, Bhushan S, and Bég O A, Colloids and Surfaces A: Physicochemical and Engineering Aspects. 506, 32 (2016)

22. Bhatti, M. M., A. Zeeshan, R. Ellahi, and N. Ijaz. Journal of Molecular Liquids 230 (2017): 237-246.

23. G C Shit, Ranjit N K, and Sinha A, Journal of Bionic Engineering. 13, 436 (2016)

24. A C Eringen, DTIC Document, 1965

25. S K Pandey and Tripathi D, Zeitschrift für Naturforschung A. 66, 181 (2011)

26. D Tripathi, Chaube M, and Gupta P, Applied Mathematics and Mechanics. 32, 1587 (2011)

27. N S Akbar and Nadeem S, Applied Nanoscience. 3, 461 (2013)

28. G Shit and Roy M, International Journal of Applied and Computational Mathematics. 1, 121 (2015)

29. G Ravi Kiran, Radhakrishnamacharya G, and Bég OA, Journal of Mechanics in Medicine and Biology. 1750013 (2016)

30. I Papautsky, et al., Sensors and actuators A: Physical. 73, 101 (1999) 
31. A A Siddiqui and Lakhtakia A, in Proceedings of the Royal Society of London A: Mathematical, Physical and Engineering Sciences. (The Royal Society, 2009), pp. 501

32. A A Siddiqui and Lakhtakia A, Journal of Physics A: Mathematical and Theoretical. 42, 355501 (2009)

33. A A Siddiqui and Lakhtakia A, Applied Mathematics and Mechanics. 34, 1305 (2013)

34. J Misra, Chandra S, and Herwig H, Journal of Hydrodynamics, Ser. B. 27, 350 (2015)

35. Z Ding, Jian Y, and Yang L, Applied Mathematics and Mechanics. 37, 769 (2016)

36. Olivares M. L., Vera-Candioti L., and Berli C. L. A., The EOF of polymer solutions, Electrophoresis 30, 921 (2009).

37. Zimmerman W., Rees J., and Craven T., Rheometry of non-Newtonian electrokinetic flow in a microchannel T-junction, Microfluid. Nanofluid. 2, 481 (2006).

38. Michael S. Bello et al. Electro-osmosis of polymer solutions in fused silica capillaries, Electrophoresis, 15, 623-626 (1994).

39. Yuki Uematsu and Takeaki Araki, Electro-osmotic flow of semidilute polyelectrolyte solutions, J. Chem. Phys. 139, 094901 (2013).

40. M. Liu and J. Yang, Electrokinetic effect of the endothelial glycocalyx layer on two-phase blood flow in small blood vessels, Microvasc. Res. 78, 14 (2009).

41. A H Shapiro, Jaffrin M Y, and Weinberg S L, Journal of Fluid Mechanics. 37, 799 (1969)

42. Hayat T, Ali N, Abbas Z, Physics Letters A. 370, 331(2007) 